\title{
EFFECTS OF TRANSPLANTING CONDITIONS AND WATER MANAGEMENT ON THE YIELD, QUALITY AND AROMA OF FRAGRANT RICE
}

\author{
TONG, T. Y. ${ }^{1,2 \#}-$ ZHANG, J. S. ${ }^{1,2 \#}-$ LI, L. ${ }^{1,2 \#}-$ LUO, H. W. ${ }^{1,2 \#}-$ HE, R. J. ${ }^{3 \#}-$ CAI, J. X. ${ }^{1}-$ MA, L. ${ }^{1,2}-$ \\ Mo, Z. W. ${ }^{1,2}-$ TANG, X. R. ${ }^{1,2^{*}}$ \\ ${ }^{I}$ Department of Crop Science and Technology, College of Agriculture, South China Agricultural \\ University, 510642 Guangzhou, PR China \\ ${ }^{2}$ Scientific Observing and Experimental Station of Crop Cultivation in South China, Ministry of \\ Agriculture, 510642 Guangzhou, PR China \\ ${ }^{3}$ College Of Agriculture, Forestry, Medicine and Technology, Hainan Radio and Tv University, \\ 570208 Hainan, PR China \\ ${ }^{\#}$ These authors have contributed equally to this work \\ *Corresponding author \\ e-mail: tangxr@scau.edu.cn; phone/fax: +20-8528-0204-618 \\ (Received $29^{\text {th }}$ Jun 2020; accepted $6^{\text {th }}$ Oct 2020)
}

\begin{abstract}
To explore the effects of transplanting conditions and water treatments on the yield, quality and aroma of fragrant rice, a field experiment was conducted with conventional fragrant rice cultivars, "Meixiangzhan 2"and "Xiangyaxiangzhan", as plant materials. Four treatments were designed: (DR) dry transplanted + rainfed, (DC) dry transplanted + conventional irrigation treatments, (WR) water transplanted + rainfed, (WC) water transplanted + conventional irrigation treatments. The highest 1000grain weight were recorded under the WR treatment, and this treatment was more beneficial to the export and accumulation of assimilates during rice growth. The average yield of the two fragrant rice cultivars in both seasons were WC $>$ WR $>$ DR $>$ DC. The milled rice rate and head rice rate were maximized in WR treatment, also minimized in chalkiness and chalky grain rate in the case of all except WC treatments in the early season. Compared with WC and DC treatments, average content of 2-acetyl-1-pyrroline (2-AP) under WR and DR treatments increased by $24.11 \%$. In conclusion, we considered WR treatment as the optimum water saving treatment for yield increase and quality improvement, as well as for increasing the aroma of fragrant rice grains.
\end{abstract}

Keywords: rice, rainfed, aroma-related enzymes, 2-acetyl-1-pyrroline, yield

\section{Introduction}

Rice (Oryza sativa L.), as an important food crop, is consumed by more than half of the world's population, and the main rice cultivars are only grown in Asia (Abid et al., 2015).

Due to its unique flavor and superior grain qualities, the fragrant rice fetches premium prices in international markets and demands are grown dramatically in recent years (Deng et al., 2018). Among the more than 100 volatile components, the aromatic compound 2-acetyl-1-pyrroline (2-AP) is the main reason for the aroma of fragrant rice (Hashemi et al., 2013). Relevant studies have confirmed that the aroma of fragrant rice is not only affected by the genetics of fragrant rice itself, but also has an important relationship with its growth and cultivation environment. The yield formation and 2-AP biosynthesis of fragrant rice were affected by many environmental factors and agronomic measures (Kong et al., 2020). In various cultivation measures, water management as an important cultivation and management measure has been concerned 
by predecessors, in which dry-wet alternative irrigation, as a new irrigation technique, has been adopted by many countries (Marchesi et al., 2019; Graham Acquaah et al., 2019). Dry-wet alternative irrigation regulated the precursors' accumulation, enzymes activities and genes expression of the enzymes that regulate the production of 2AP in both rice cultivars (Bao et al., 2018a).

Related studies have shown that light water-controlled irrigation can increase the aroma content of fragrant rice grains and decrease the chalky grain rate, and it is the best cultivation measure for high yield and high quality of fragrant rice (Tian et al., 2018). Strong water stress affected the physiological activity of rice in the later stage and reduced the synthesis and accumulation of 2-AP in fragrant rice, so water had a great influence on the aroma of fragrant rice (Wang et al., 2013).

Predecessors had a detailed description of intermittent irrigation and water-saving irrigation, but there were few reports on the effects of water treatment before sowing and the whole cultivation process of fragrant rice on the yield, quality and aroma of fragrant rice. To explore the differences in yield, quality and aroma of fragrant rice, two fragrant rice cultivars, "Meixiangzhan-2"and "Xiangyaxiangzhan", which were widely planted in South China, were used as experimental materials. The purpose of this study is to provide a theoretical basis for high yield, quality and more aroma of fragrant rice.

\section{Materials and methods}

\section{Experimental details}

A two-season field experiment was conducted in the Experimental Research Farm, College of Agriculture, South China Agricultural University, Guangzhou $\left(23^{\circ} 09^{\prime} \mathrm{N}\right.$, $113^{\circ} 22^{\prime} \mathrm{E}$ and $11 \mathrm{~m}$ from mean sea level), in China in 2019. The physical and chemical properties of soil in the early season were as follows: $\mathrm{pH} 6.5$, organic matter content $22.63 \mathrm{~g} / \mathrm{kg}$, total nitrogen $1.56 \mathrm{~g} / \mathrm{kg}$, total phosphorus $1.43 \mathrm{~g} / \mathrm{kg}$, total potassium $23.97 \mathrm{~g} / \mathrm{kg}$, available potassium $90.32 \mathrm{mg} / \mathrm{kg}$, available phosphorus $75.70 \mathrm{mg} / \mathrm{kg}$; Physical and chemical properties of soil in late season: $\mathrm{pH} 6.2$, organic matter content $25.63 \mathrm{~g} / \mathrm{kg}$, total nitrogen $1.66 \mathrm{~g} / \mathrm{kg}$, total phosphorus $1.57 \mathrm{~g} / \mathrm{kg}$, total potassium $22.83 \mathrm{~g} / \mathrm{kg}$, available potassium $92.32 \mathrm{mg} / \mathrm{kg}$, available phosphorus $45.63 \mathrm{mg} / \mathrm{kg}$.

Two fragrant rice cultivars, "Meixiangzhan 2" (Lemont $\times$ Fengaozhan) and "Xiangyaxiangzhan" (Xiangsimiao 126× Xiangyaruanzhan), were used as materials in the experiment. Those cultivars are well-known and widely grown in South China. The split zone test design is adopted in the experiment.

The soil conditions at transplanting were set as: dry transplanted treatment (D): the former cropping was not soaked after harvest. 5 days after harvest, rotary tillage machine was used for dry rotary tillage once and the second dry rotary tillage was carried out 3 days before transplanting rice seedlings. Water transplanted treatment (W): irrigation was carried out 3-4 cm in the field 10 days before transplanting. After keeping water for 3 days, the rotary tillage was used to rotate once. The water treatments were set as: rainfed (R), if there is no wilting of the seedlings after transplanting, no artificial irrigation was carried out. If wilting occurred, irrigation was carried out to ensure that the rice would not die. In conventional irrigation treatments $(\mathrm{C})$ shallow water was maintained at the early stage, then drained and exposed the field after enough seedlings, $3-4 \mathrm{~cm}$ irrigation was carried out at the beginning of young panicle differentiation, then kept dry and wet alternately from booting stage to breaking stage, then kept in shallow water layer after heading to facilitate filling and fruiting, and water was cut off 5-6 days before harvest. The plot sizes of the four 
treatments were $10.5 \times 7 \mathrm{~m}^{2}$. Each treatment special fertilizer was applied for fragrant rice $\left(\mathrm{N}+\mathrm{P}_{2} \mathrm{O}_{5}+\mathrm{K}_{2} \mathrm{O} \geq 6 \%\right.$, organic matter $\left.\geq 25 \%\right) 750 \mathrm{~kg} / \mathrm{hm}^{2}$ at the tillering stage, and ridges were built between plots ( $40 \mathrm{~cm}$ wide and wrapped in plastic) to keep water and fertilizer. The seeds of both rice cultivars were surface sterilized with a 5\% sodium hypochlorite solution and then immersed in water for $12 \mathrm{~h}$ and allowed to germinate in a dark thermostatic incubator at $38^{\circ} \mathrm{C}$ for $12 \mathrm{~h}$. The germinated seeds were sown in PVC trays for nursery raising, then, PVC trays were placed in puddled field and covered with a plastic sheet. 15-day-old seedlings were transplanted to the field. Geminated seeds of both cultivars were sown on the 9th March, 2019 (early season) and the 15th July 2019 (late season) for nursery rising. Seedlings were transplanted in puddled field on the 26th March and the 2nd August, 2019 for early and late season, respectively, and harvested on 14th July (early season) and 7th November (late season) (Fig. 1).

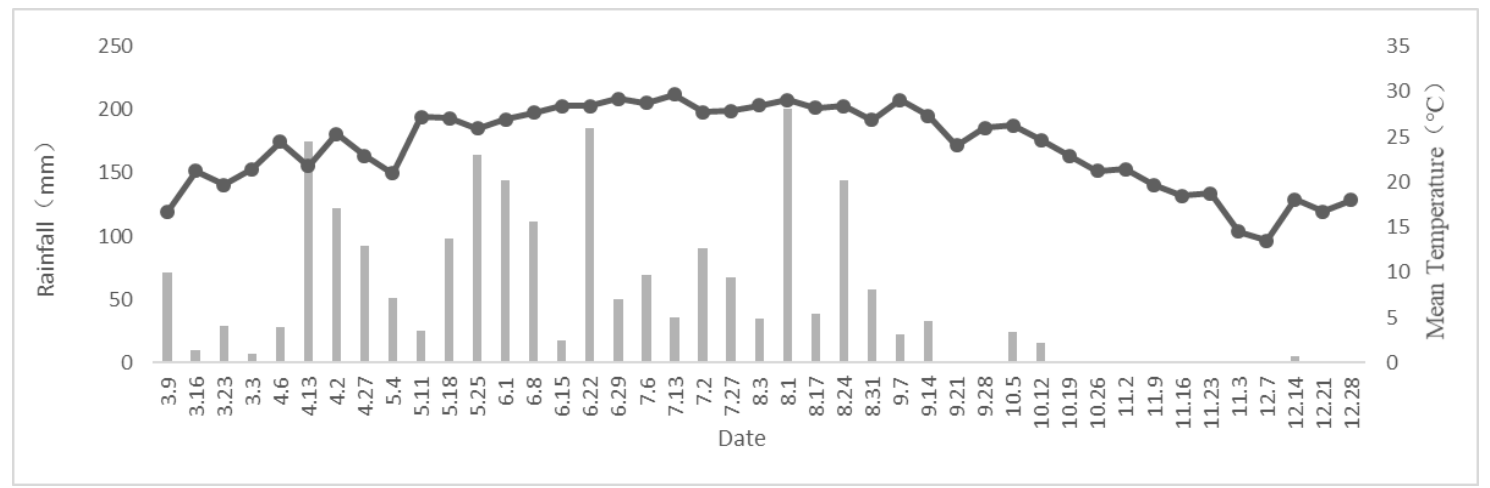

Figure 1. Mean weekly temperature and rainfall during the experiment

\section{Treatments and plant sampling}

Experimental treatments were carried out as described:

- DR: dry transplanted + rainfed,

- DC: dry transplanted + conventional irrigation treatments,

- WR: water transplanted + rainfed,

- WC: water transplanted + conventional irrigation treatments.

Nine random rice plants from each plot were collected for estimation of accumulation and assimilation of photosynthetic matter, aroma-related enzymes and 2-acetyl-1-pyrroline (2-AP) in grains in the tillering stage (early season April on 26th, late season on September 5th), booting stage (early season on the 19th May, late season on the 24th September), full heading stage (early season on the 11th June, late season on the 10th October), 15 days after heading (early season on the 26th June, late season on the $25^{\text {th }}$ October), and mature stage (early season on the $4^{\text {th }}$ July, late season on the $13^{\text {th }}$ November).

\section{Accumulation and assimilation of photosynthetic matter}

The leaf area was measured according to the methods of Tao et al. (2006). According to the average tiller number of the community, 6 hills of rice plants with the same growth pattern were taken. The leaf area of rice samples was measured by the product of length and width of 15 flag leaves. Then the tissues of different parts of rice were cut and dried at $105^{\circ} \mathrm{C}$ for $30 \mathrm{~min}$ and dried in oven at $80^{\circ} \mathrm{C}$ for $30 \mathrm{~min}$. The dry matter 
mass was measured to determine the community growth rate (CGR) and net assimilation rate (NAR).

$$
\begin{gathered}
\operatorname{CGR}\left(\mathrm{g} /\left(\mathrm{m}^{2} \cdot \mathrm{d}\right)\right)=(W 2-W 1) /(t 2-t 1) \\
\operatorname{NAR}\left(\mathrm{g} /\left(\mathrm{m}^{2} \cdot \mathrm{d}\right)\right)=[(\operatorname{lnLAI} 2-\operatorname{lnLAI} 1) /(\operatorname{LAI} 2-\operatorname{LAI} 1)] \times[(W 2-W 1) /(t 2-t 1)]
\end{gathered}
$$

where LAI1 and LAI2 are the leaf area index measured before and after, $\mathrm{t} 1$ and $\mathrm{t} 2$ are the time of the two measurements, and $\mathrm{W} 1$ and $\mathrm{W} 2$ are the dry matter mass measured before and after the two measurements.

\section{Aroma-related enzymes and 2-acetyl-1-pyrroline (2-AP) in grains}

According to the average number of tillers of the population, 3 hills of rice samples with the same growth were taken back to the laboratory, the tissues of different parts (including the leaves, stems and grains) of rice were quickly separated and placed in liquid nitrogen. The leaves, stems and grains were placed in the refrigerator at $-80{ }^{\circ} \mathrm{C}$.

The determination method of proline refers to the method of Huang et al. (2012). The contents of pyrroline-5-carboxylic acid (P5C) were measured according to the method of Luo et al. (2019). Ornithine aminotransferase (OAT) activity was assayed according to the method devised by Bao et al. (2018b), the measurement of methylglyoxal refers to the method of Hasanuzzaman et al. (2019), and the determination method of aroma (2-AP) in grains refers to the method of $\mathrm{Du}$ et al. (2019). The measurements were repeated in triplicate and averaged.

\section{Measurement of yield and yield-related traits}

One community sample area $\left(1 \mathrm{~m}^{2}\right)$ from each experimental plot was harvested at maturity, then threshed manually and sun-dried. 30 random hills of rice plants in each plot were sampled for calculating the average effective panicle number per hill. The above measures have been repeated thrice. According to the average number of effective panicles, 5 representative plants were randomly selected to determine the number of grain count per panicle, seed setting rate and 1000-grain weight.

\section{Measurement of grain quality}

After sun-dried, grains were stored at room temperature for at least three months to determine grain quality attributes. Rice grains from each treatment was taken from storage and brown rice rate was estimated using a rice huller (Jiangsu, China) while milled rice and head rice recovery rates were calculated by using a Jingmi testing rice grader (Zhejiang, China). Grains with chalkiness and chalky grain rate were estimated by using an SDE-A light box (Guangzhou, China) while an Infratec-1241 grain analyzer (FOSS-TECATOR) was used to determine the grain amylose and protein contents.

\section{Statistical analyses}

All statistical analyses were performed by using Statistix 8 (Analytical software, Tallahassee, Florida, USA). Two factors (transplanting conditions and water management) and their interactions had been tested. The data were analyzed by one- 
way analysis of variance and the differences amongst means were separated by using least significant difference (LSD) test at 5\% significance level.

\section{Results}

\section{Community growth rate and net assimilation rate}

As shown in Table 1, for Meixiangzhan 2, the highest community growth rate (CGR) was recorded under the WR treatment, in all except for WC treatment from booting stage to 15 days after heading in the early season, and the trend of net assimilation rate (NAR) from booting stage and 15 days after heading in early season were the same: $\mathrm{WR}>\mathrm{WC}=\mathrm{DC}>\mathrm{DR}$. In late season, the CGR under WR treatment was the highest at tillering stage, and the NAR had the same trend. For Xiangyaxiangzhan, there was no significant regular change in the treatments in the early season, and the CGR was the highest under WR treatment at tillering stage, and the NAR under WR treatment was also significantly higher than that of DC and WC treatment at tillering stage.

Table 1. Effects of transplanting conditions and water management on CGR and NAR of fragrant rice

\begin{tabular}{|c|c|c|c|c|c|c|c|c|}
\hline \multirow{2}{*}{ Season } & \multirow{2}{*}{ Cultivar } & \multirow{2}{*}{ Treatment } & \multicolumn{3}{|c|}{$\mathrm{CGR} /\left(\mathrm{g} \cdot \mathrm{m}^{-2} \cdot \mathrm{d}^{-1}\right)$} & \multicolumn{3}{|c|}{$\mathrm{NAR} /\left(\mathrm{g} \cdot \mathrm{m}^{-2} \cdot \mathrm{d}^{-1}\right)$} \\
\hline & & & T-B & $\mathbf{B}-\mathbf{H}$ & H-HA & T-B & $\mathbf{B}-\mathbf{H}$ & H-HA \\
\hline \multirow{8}{*}{ Early } & \multirow{4}{*}{ Meixiangzhan 2} & DR & $14.23 \pm 2.59 \mathrm{~b}$ & $10.53 \pm 2.51 \mathrm{~b}$ & $8.89 \pm 2.99 \mathrm{~b}$ & $4.74 \pm 1.36 \mathrm{~b}$ & $1.98 \pm 0.47 \mathrm{~b}$ & $2.21 \pm 0.74 b$ \\
\hline & & DC & $15.43 \pm 1.89 \mathrm{~b}$ & $8.36 \pm 3.39 \mathrm{~b}$ & $10.76 \pm 2.91 \mathrm{ab}$ & $4.83 \pm 1.13 b$ & $2.38 \pm 1.51 \mathrm{ab}$ & $2.83 \pm 0.76 \mathrm{ab}$ \\
\hline & & WR & $14.27 \pm 3.45 \mathrm{~b}$ & $17.98 \pm 0.31 \mathrm{a}$ & $14.09 \pm 3.29 \mathrm{a}$ & $3.69 \pm 1.46 \mathrm{c}$ & $3.72 \pm 0.06 \mathrm{a}$ & $3.08 \pm 0.72 \mathrm{a}$ \\
\hline & & WC & $21.55 \pm 2.52 \mathrm{a}$ & $23.41 \pm 3.19 \mathrm{a}$ & $10.76 \pm 2.15 \mathrm{ab}$ & $5.28 \pm 1.31 \mathrm{a}$ & $2.44 \pm 0.6 \mathrm{ab}$ & $2.42 \pm 0.48 \mathrm{ab}$ \\
\hline & \multirow{4}{*}{ Xiangyaxiangzhan } & DR & $14.17 \pm 3.45 \mathrm{ab}$ & $8.24 \pm 4.85 \mathrm{c}$ & $9.43 \pm 4.44 \mathrm{~b}$ & $5.32 \pm 1.32 \mathrm{a}$ & $1.98 \pm 0.98 \mathrm{~b}$ & $2.74 \pm 1.05 \mathrm{a}$ \\
\hline & & DC & $11.61 \pm 2.58 \mathrm{~b}$ & $25.87 \pm 3.28 \mathrm{a}$ & $3.49 \pm 0.89 \mathrm{c}$ & $4.53 \pm 1.41 \mathrm{a}$ & $4.87 \pm 1.29 \mathrm{a}$ & $1.35 \pm 0.77 \mathrm{~b}$ \\
\hline & & WR & $17.32 \pm 1.18 \mathrm{a}$ & $9.82 \pm 0.14 \mathrm{c}$ & $9.09 \pm 4.32 \mathrm{~b}$ & $5.75 \pm 0.39 \mathrm{a}$ & $3.85 \pm 1.47 \mathrm{ab}$ & $2.17 \pm 0.36 \mathrm{a}$ \\
\hline & & WC & $12.29 \pm 1.59 \mathrm{~b}$ & $17.11 \pm 2.26 \mathrm{~b}$ & $18.26 \pm 4.65 \mathrm{a}$ & $4.55 \pm 1.01 \mathrm{a}$ & $4.62 \pm 1.47 \mathrm{a}$ & $2.23 \pm 1.32 \mathrm{a}$ \\
\hline \multirow{8}{*}{ Late } & \multirow{4}{*}{ Meixiangzhan 2} & DR & $12.61 \pm 1.17 \mathrm{ab}$ & $21.59 \pm 0.26 \mathrm{a}$ & $13.48 \pm 1.97 \mathrm{~b}$ & $5.21 \pm 0.68 \mathrm{a}$ & $5.08 \pm 0.08 \mathrm{~b}$ & $3.69 \pm 0.65 \mathrm{a}$ \\
\hline & & DC & $13.16 \pm 2.59 \mathrm{ab}$ & $22.21 \pm 0.56 \mathrm{a}$ & $16.21 \pm 4.41 \mathrm{a}$ & $3.74 \pm 0.74 b$ & $6.82 \pm 1.04 \mathrm{a}$ & $3.05 \pm 0.51 \mathrm{a}$ \\
\hline & & WR & $16.56 \pm 1.44 \mathrm{a}$ & $15.26 \pm 4.92 \mathrm{~b}$ & $13.57 \pm 4.55 \mathrm{~b}$ & $5.01 \pm 0.44 \mathrm{a}$ & $2.62 \pm 0.51 \mathrm{c}$ & $3.31 \pm 0.94 \mathrm{a}$ \\
\hline & & WC & $10.56 \pm 4.54 \mathrm{~b}$ & $20.42 \pm 1.62 \mathrm{a}$ & $17.58 \pm 2.81 \mathrm{a}$ & $2.31 \pm 0.71 \mathrm{c}$ & $3.92 \pm 1.05 \mathrm{~b}$ & $3.37 \pm 0.71 \mathrm{a}$ \\
\hline & \multirow{4}{*}{ Xiangyaxiangzhan } & DR & $19.13 \pm 4.79 a$ & $9.98 \pm 2.24 \mathrm{c}$ & $16.03 \pm 4.36 \mathrm{~b}$ & $4.27 \pm 0.73 \mathrm{a}$ & $2.19 \pm 0.55 \mathrm{c}$ & $3.28 \pm 1.23 b$ \\
\hline & & DC & $18.56 \pm 4.96 \mathrm{ab}$ & $8.28 \pm 4.74 \mathrm{c}$ & $20.01 \pm 2.62 b$ & $4.01 \pm 1.07 \mathrm{a}$ & $3.41 \pm 1.11 b c$ & $3.24 \pm 1.33 \mathrm{~b}$ \\
\hline & & WR & $12.86 \pm 1.98 \mathrm{bc}$ & $26.23 \pm 0.55 \mathrm{a}$ & $30.78 \pm 0.93 \mathrm{a}$ & $4.13 \pm 0.41 \mathrm{a}$ & $5.23 \pm 0.94 \mathrm{a}$ & $4.18 \pm 1.67 \mathrm{a}$ \\
\hline & & WC & $9.48 \pm 3.39 \mathrm{c}$ & $14.6 \pm 2.06 \mathrm{~b}$ & $19.59 \pm 3.85 \mathrm{~b}$ & $2.52 \pm 0.14 \mathrm{~b}$ & $3.77 \pm 0.74 \mathrm{ab}$ & $3.22 \pm 0.27 \mathrm{~b}$ \\
\hline
\end{tabular}

(DR) dry transplanted + rained irrigation, (DC) dry transplanted + conventional irrigation treatments, (WR) water transplanted + rainfed irrigation, (WC) water transplanted + conventional irrigation treatments. T-B: Tillering stage to booting stage, B-H: Booting stage to heading stage, H-HA: Heading stage to Heading after 15 days. Small alphabetical letters above means indicate the differences $(\mathrm{P}<0.05)$ among different management treatments

\section{Yield and yield-related traits}

As shown in Table 2, for Meixiangzhan 2, The trend of fertile panicle was: $\mathrm{WR}=\mathrm{WC}>\mathrm{DR}=\mathrm{DC}$ in both early and late season. Also the highest 1000-grain weight was recorded with WR treatment in the early season. For Xiangyaxiangzhan, in the early season, the trend of fertile panicle was the same as Meixiangzhan 2. Compared with $\mathrm{C}$ treatments, $\mathrm{R}$ 
treatments could also increase the 1000-grain weight of these cultivars, and the highest yield was recorded under the WR treatment in the early season. The varieties and the experiments did not influence the yield significantly, so the average yield of the two fragrant rice cultivars in both seasons were WC $>$ WR $>$ DR $>$ DC.

Table 2. Effects of transplanting conditions and water management yield and yield-related traits

\begin{tabular}{|c|c|c|c|c|c|c|c|}
\hline Season & Cultivar & Treatment & $\begin{array}{c}\text { Fertile panicle } \\
\left(\mathbf{m}^{-1}\right)\end{array}$ & $\begin{array}{c}\text { Grain count per } \\
\text { panicle }\end{array}$ & $\begin{array}{c}\text { Seed setting rate } \\
(\%)\end{array}$ & $\begin{array}{l}\text { 1000-grain } \\
\text { weight (g) }\end{array}$ & $\begin{array}{l}\text { Actual yield } \\
\left(\mathrm{t} \mathrm{ha}^{-1}\right)\end{array}$ \\
\hline \multirow{8}{*}{ Early } & \multirow{4}{*}{ Meixiangzhan 2} & DR & $257.11 \pm 0.43 b$ & $131.39 \pm 6.89 b$ & $82.63 \pm 3.92 b$ & $18.80 \pm 0.60 \mathrm{ab}$ & $3.25 \pm 0.28 b$ \\
\hline & & DC & $252.33 \pm 1.22 b$ & $151.90 \pm 5.72 \mathrm{a}$ & $82.57 \pm 8.33 b$ & $17.55 \pm 1.25 b$ & $3.33 \pm 0.11 b$ \\
\hline & & WR & $261.42 \pm 0.45 \mathrm{a}$ & $148.32 \pm 8.29 \mathrm{a}$ & $87.91 \pm 3.46 \mathrm{a}$ & $19.36 \pm 1.52 \mathrm{a}$ & $3.48 \pm 0.21 \mathrm{a}$ \\
\hline & & WC & $260.22 \pm 0.22 \mathrm{a}$ & $153.04 \pm 2.59 \mathrm{a}$ & $86.91 \pm 5.51 \mathrm{a}$ & $18.82 \pm 0.72 \mathrm{ab}$ & $3.23 \pm 0.43 b$ \\
\hline & \multirow{4}{*}{ Xiangyaxiangzhan } & DR & $252.22 \pm 0.11 b$ & $124.94 \pm 3.63 b$ & $82.81 \pm 5.17 \mathrm{ab}$ & $20.44 \pm 0.62 \mathrm{a}$ & $2.72 \pm 0.49 b$ \\
\hline & & $\mathrm{DC}$ & $252.12 \pm 1.22 b$ & $129.83 \pm 8.73 b$ & $79.95 \pm 1.36 b$ & $20.50 \pm 0.40 \mathrm{a}$ & $2.97 \pm 0.11 \mathrm{ab}$ \\
\hline & & WR & $275.94 \pm 0.23 a$ & $142.16 \pm 6.16 \mathrm{a}$ & $85.31 \pm 2.42 \mathrm{a}$ & $19.66 \pm 0.36 \mathrm{ab}$ & $3.55 \pm 0.43 \mathrm{a}$ \\
\hline & & WC & $272.77 \pm 0.32 \mathrm{a}$ & $133.30 \pm 3.02 b$ & $86.42 \pm 2.44 a$ & $19.01 \pm 1.40 \mathrm{~b}$ & $3.35 \pm 0.28 \mathrm{ab}$ \\
\hline \multirow{8}{*}{ Late } & \multirow{4}{*}{ Meixiangzhan 2} & DR & $246.13 \pm 1.22 b$ & $140.27 \pm 19.13 \mathrm{ab}$ & $89.12 \pm 1.03 a$ & $19.11 \pm 1.22 b$ & $5.68 \pm 0.49 a$ \\
\hline & & DC & $242.22 \pm 0.32 b$ & $152.75 \pm 23.16 a$ & $86.89 \pm 0.03 a$ & $20.79 \pm 1.23 \mathrm{a}$ & $4.64 \pm 0.23 b$ \\
\hline & & WR & $276.58 \pm 1.01 \mathrm{a}$ & $122.45 \pm 15.35 b$ & $90.13 \pm 2.02 \mathrm{a}$ & $19.21 \pm 0.21 b$ & $4.80 \pm 0.52 b$ \\
\hline & & WC & $270.13 \pm 0.31 \mathrm{a}$ & $137.95 \pm 20.83 \mathrm{ab}$ & $88.32 \pm 0.05 a$ & $20.21 \pm 0.93 \mathrm{ab}$ & $5.52 \pm 0.68 \mathrm{a}$ \\
\hline & \multirow{4}{*}{ Xiangyaxiangzhan } & DR & $255.12 \pm 1.21 b$ & $138.86 \pm 11.29 \mathrm{a}$ & $84.24 \pm 1.06 \mathrm{~b}$ & $18.88 \pm 0.57 \mathrm{~b}$ & $5.52 \pm 0.39 b$ \\
\hline & & $\mathrm{DC}$ & $268.21 \pm 0.22 \mathrm{ab}$ & $130.70 \pm 5.74 b$ & $83.33 \pm 0.36 b$ & $20.28 \pm 0.44 a$ & $5.62 \pm 0.30 \mathrm{a}$ \\
\hline & & WR & $285.54 \pm 1.11 \mathrm{a}$ & $143.27 \pm 6.20 \mathrm{a}$ & $88.29 \pm 1.24 \mathrm{ab}$ & $20.04 \pm 0.66 \mathrm{a}$ & $5.68 \pm 1.19 a$ \\
\hline & & WC & $281.12 \pm 1.65 \mathrm{a}$ & $125.63 \pm 14.95 b$ & $91.25 \pm 0.33 \mathrm{a}$ & $18.96 \pm 1.55 b$ & $5.42 \pm 0.85 c$ \\
\hline \multirow{3}{*}{ Early } & \multirow{3}{*}{\multicolumn{2}{|c|}{$\begin{array}{l}\mathrm{C} \\
\mathrm{T}\end{array}$}} & 1.43 & 1.43 & $13.36^{*}$ & 1.25 & 3.94 \\
\hline & & & 0.03 & 0.03 & $12.18^{*}$ & 2.47 & 1.05 \\
\hline & & & 1.66 & 1.66 & $11.17^{*}$ & 0.34 & 3.64 \\
\hline \multirow{3}{*}{ Late } & \multirow{2}{*}{\multicolumn{2}{|c|}{$\begin{array}{l}\mathrm{C} \\
\mathrm{T}\end{array}$}} & $16.03 *$ & $5.28 *$ & 1.46 & 0.81 & 3.19 \\
\hline & & & $15.03 *$ & $12.22 *$ & 2.62 & 2.42 & 0.81 \\
\hline & \multicolumn{2}{|l|}{$\mathrm{C} \times \mathrm{T}$} & $5.01 *$ & $20.86^{*}$ & 2.01 & 1.65 & 1.74 \\
\hline
\end{tabular}

(DR) dry transplanted + rained irrigation, (DC) dry transplanted + conventional irrigation treatments, (WR) water transplanted + rainfed irrigation, (WC) water transplanted + conventional irrigation treatments. Small alphabetical letters above means indicate the differences $(\mathrm{P}<0.05)$ among different management treatments. *, ** Significantly different at 0.05 and 0.01 probability levels, respectively. C: Cultivar; T: Treatment; $\mathrm{C} \times \mathrm{T}$ : Cultivar- Treatment interaction. The same as below

\section{Quality of fragrant rice}

As shown in Table 3, for Meixiangzhan 2, there was no significant difference among the four treatments in the early season on the rate of brown rice and milled rice of this cultivar. The head rice rate maximized in WR treatment. And maximized in DR treatment in the early and late season. For Xiangyaxiangzhan, WR treatment had the highest milled rice rate and head rice rate and the lowest chalkiness and chalky grain rate in all except WC treatments in the early season, while the four treatments had no significant effect on the brown rice rate and milled rice rate of this cultivar in the late season. Each of the treatments had little effect on the length-width ratio of the two fragrant rice cultivars.

\section{Proline content}

The effects of different transplanting conditions and water management on the proline content in the leaves of fragrant rice was shown in Table 4. For Meixiangzhan, 
the trend of proline contents was: $\mathrm{DR}=\mathrm{DC}>\mathrm{WR}=\mathrm{WC}$ at tillering stage and booting stage. And the proline content in leaves had the same trend in late season. For Xiangyaxiangzhan, DR and DC treatments maximized the leaf proline content of this cultivar at four stages (except heading after 15 days) in the early season, and the trend in the late season was similar. 
Table 3. Effects of transplanting conditions and water management on quality of fragrant rice

\begin{tabular}{|c|c|c|c|c|c|c|c|c|c|c|c|}
\hline Season & Cultivar & Treatment & \begin{tabular}{|c|} 
Brown rice rate \\
$(\%)$
\end{tabular} & \begin{tabular}{|c|} 
Milled rice rate \\
$(\%)$
\end{tabular} & \begin{tabular}{|c|} 
Head rice rate \\
$(\%)$
\end{tabular} & Protein & Amylose & Akali & Chalkiness (\%) & \begin{tabular}{|c|} 
Chalky grain rate \\
$(\%)$
\end{tabular} & $\begin{array}{c}\text { The ratio of length to } \\
\text { width }(\%)\end{array}$ \\
\hline \multirow{8}{*}{ Early } & \multirow{4}{*}{ Meixiangzhan 2} & $\mathrm{DR}$ & $74.19 \pm 1.69 \mathrm{a}$ & $61.09 \pm 1.57 \mathrm{a}$ & $55.76 \pm 1.35 \mathrm{ab}$ & $7.43 \pm 0.05 \mathrm{a}$ & $19.97 \pm 0.69 \mathrm{a}$ & $5.61 \pm 0.08 \mathrm{~b}$ & $1.45 \pm 0.11 \mathrm{~b}$ & $5.05 \pm 1.13 b$ & $2.92 \pm 0.19 \mathrm{a}$ \\
\hline & & DC & $74.01 \pm 0.49 \mathrm{a}$ & $64.22 \pm 0.87 \mathrm{a}$ & $52.26 \pm 1.14 \mathrm{~b}$ & $6.17 \pm 0.09 \mathrm{~d}$ & $19.67 \pm 0.12 \mathrm{ab}$ & $5.53 \pm 0.05 b c$ & $1.46 \pm 0.03 b$ & $5.12 \pm 2.29 b$ & $2.77 \pm 0.05 \mathrm{ab}$ \\
\hline & & WR & $76.16 \pm 0.21 \mathrm{a}$ & $63.52 \pm 0.43 \mathrm{a}$ & $57.98 \pm 1.06 \mathrm{a}$ & $6.87 \pm 0.05 \mathrm{~b}$ & $18.60 \pm 0.51 b$ & $5.90 \pm 0.10 \mathrm{a}$ & $2.39 \pm 0.65 \mathrm{a}$ & $5.63 \pm 3.35 \mathrm{a}$ & $2.66 \pm 0.12 b$ \\
\hline & & WC & $75.71 \pm 0.08 \mathrm{a}$ & $66.05 \pm 1.14 \mathrm{a}$ & $57.23 \pm 1.66 \mathrm{a}$ & $6.37 \pm 0.05 \mathrm{c}$ & $19.80 \pm 0.71 \mathrm{a}$ & $5.37 \pm 0.12 c$ & $1.58 \pm 0.18 b$ & $5.17 \pm 3.26 \mathrm{ab}$ & $2.81 \pm 0.11 \mathrm{ab}$ \\
\hline & \multirow{4}{*}{ Xiangyaxiangzhan } & DR & $73.13 \pm 0.09 \mathrm{a}$ & $58.52 \pm 1.85 \mathrm{~b}$ & $55.93 \pm 1.19 \mathrm{ab}$ & $7.33 \pm 0.05 \mathrm{a}$ & $17.30 \pm 0.67 \mathrm{a}$ & $5.73 \pm 0.05 \mathrm{a}$ & $1.06 \pm 0.63 \mathrm{ab}$ & $4.35 \pm 3.22 b$ & $3.14 \pm 0.21 \mathrm{a}$ \\
\hline & & DC & $72.58 \pm 0.42 \mathrm{a}$ & $62.46 \pm 0.24 \mathrm{ab}$ & $54.63 \pm 0.21 \mathrm{ab}$ & $7.07 \pm 0.05 \mathrm{c}$ & $18.50 \pm 1.28 \mathrm{a}$ & $5.63 \pm 0.05 \mathrm{ab}$ & $2.08 \pm 0.89 \mathrm{a}$ & $5.66 \pm 3.84 \mathrm{a}$ & $3.03 \pm 0.22 \mathrm{a}$ \\
\hline & & WR & $72.49 \pm 3.35 \mathrm{a}$ & $63.25 \pm 0.86 \mathrm{a}$ & $56.52 \pm 0.72 \mathrm{a}$ & $7.17 \pm 0.05 \mathrm{bc}$ & $18.93 \pm 0.05 \mathrm{a}$ & $5.63 \pm 0.05 \mathrm{ab}$ & $0.75 \pm 0.09 b$ & $3.69 \pm 2.26 \mathrm{c}$ & $2.84 \pm 0.14 \mathrm{a}$ \\
\hline & & WC & $72.94 \pm 0.48 \mathrm{a}$ & $61.88 \pm 0.37 \mathrm{ab}$ & $53.51 \pm 0.46 \mathrm{~b}$ & $7.23 \pm 0.05 \mathrm{ab}$ & $17.10 \pm 0.90 \mathrm{a}$ & $5.60 \pm 0.08 \mathrm{~b}$ & $1.49 \pm 0.77 \mathrm{ab}$ & $4.41 \pm 3.13 b$ & $3.14 \pm 0.15 \mathrm{a}$ \\
\hline \multirow{8}{*}{ Late } & \multirow{4}{*}{ Meixiangzhan 2} & $\mathrm{DR}$ & $76.76 \pm 0.21 \mathrm{a}$ & $67.48 \pm 0.91 \mathrm{a}$ & $65.22 \pm 0.85 \mathrm{a}$ & $8.27 \pm 0.12 \mathrm{a}$ & $19.13 \pm 0.54 \mathrm{a}$ & $6.67 \pm 0.12 \mathrm{a}$ & $0.61 \pm 0.69 c$ & $3.12 \pm 2.66 \mathrm{~b}$ & $2.67 \pm 0.12 \mathrm{a}$ \\
\hline & & DC & $76.66 \pm 0.35 a$ & $65.62 \pm 0.88 \mathrm{a}$ & $62.19 \pm 0.57 \mathrm{~b}$ & $7.97 \pm 0.12 \mathrm{~b}$ & $19.07 \pm 0.41 \mathrm{a}$ & $6.72 \pm 0.08 \mathrm{a}$ & $0.78 \pm 0.24 b$ & $3.37 \pm 5.36 \mathrm{~b}$ & $2.74 \pm 0.11 \mathrm{a}$ \\
\hline & & WR & $76.58 \pm 0.69 \mathrm{a}$ & $65.56 \pm 1.05 \mathrm{a}$ & $63.24 \pm 1.34 \mathrm{ab}$ & $7.81 \pm 0.08 \mathrm{~b}$ & $19.51 \pm 0.43 \mathrm{a}$ & $6.63 \pm 0.12 \mathrm{a}$ & $1.06 \pm 0.68 \mathrm{a}$ & $4.54 \pm 5.21 \mathrm{a}$ & $2.72 \pm 0.09 \mathrm{a}$ \\
\hline & & WC & $76.86 \pm 0.11 \mathrm{a}$ & $66.66 \pm 0.48 \mathrm{a}$ & $63.66 \pm 0.48 \mathrm{ab}$ & $8.41 \pm 0.08 \mathrm{a}$ & $18.77 \pm 0.12 \mathrm{a}$ & $6.73 \pm 0.17 \mathrm{a}$ & $0.79 \pm 0.23 b$ & $3.41 \pm 5.71 b$ & $2.71 \pm 0.13 \mathrm{a}$ \\
\hline & \multirow{4}{*}{ Xiangyaxiangzhan } & DR & $75.36 \pm 0.37 \mathrm{a}$ & $62.03 \pm 0.54 \mathrm{a}$ & $56.32 \pm 0.21 \mathrm{~b}$ & $8.21 \pm 0.08 \mathrm{a}$ & $18.70 \pm 0.08 \mathrm{~b}$ & $6.83 \pm 0.12 \mathrm{a}$ & $0.58 \pm 0.48 \mathrm{ab}$ & $4.13 \pm 2.82 \mathrm{a}$ & $2.67 \pm 0.12 \mathrm{a}$ \\
\hline & & DC & $74.91 \pm 0.49 \mathrm{a}$ & $62.65 \pm 1.64 \mathrm{a}$ & $55.06 \pm 1.62 \mathrm{~b}$ & $7.97 \pm 0.12 \mathrm{ab}$ & $19.63 \pm 0.05 \mathrm{a}$ & $6.67 \pm 0.12 \mathrm{a}$ & $1.23 \pm 0.66 \mathrm{a}$ & $3.76 \pm 2.87 \mathrm{ab}$ & $2.77 \pm 0.09 \mathrm{a}$ \\
\hline & & WR & $75.05 \pm 0.81 \mathrm{a}$ & $63.76 \pm 1.16 \mathrm{a}$ & $59.57 \pm 0.88 \mathrm{a}$ & $7.92 \pm 0.08 \mathrm{~b}$ & $18.92 \pm 0.22 b$ & $6.67 \pm 0.12 \mathrm{a}$ & $0.16 \pm 0.06 \mathrm{~b}$ & $3.59 \pm 2.68 b$ & $2.81 \pm 0.11 \mathrm{a}$ \\
\hline & & WC & $75.55 \pm 0.69 \mathrm{a}$ & $64.51 \pm 1.47 \mathrm{a}$ & $59.84 \pm 1.13 a$ & $7.97 \pm 0.12 \mathrm{ab}$ & $18.21 \pm 0.36 \mathrm{c}$ & $6.63 \pm 0.12 \mathrm{a}$ & $0.55 \pm 0.11 \mathrm{ab}$ & $3.45 \pm 1.49 b$ & $2.92 \pm 0.21 \mathrm{a}$ \\
\hline \multirow{3}{*}{ Early } & \multicolumn{2}{|l|}{$\mathrm{C}$} & $8.44 * *$ & $9.75^{* *}$ & 2.51 & $16.45^{* * *}$ & $20.61 * *$ & 2.12 & 3.01 & $23.788 * *$ & $15.98^{* * *}$ \\
\hline & \multicolumn{2}{|l|}{$\mathrm{T}$} & 2.04 & 1.79 & $14.68^{* *}$ & $12.15^{* *}$ & $4.53^{*}$ & $12.31^{* *}$ & 0.92 & $6.99 * *$ & $3.67^{*}$ \\
\hline & \multicolumn{2}{|l|}{$\mathrm{C} * \mathrm{~T}$} & 0.72 & 2.589 & $8.52 * *$ & $15.73^{* *}$ & $16.95^{* *}$ & $10.12^{* *}$ & $4.738^{*}$ & $19.58^{* *}$ & 0.28 \\
\hline \multirow{3}{*}{ Late } & \multicolumn{2}{|l|}{$\mathrm{C}$} & $11.18^{* *}$ & $12.43^{* * *}$ & $35.46^{* * *}$ & 3.6 & 2.52 & 0.07 & 0.85 & 0.11 & 2.25 \\
\hline & \multicolumn{2}{|l|}{$\mathrm{T}$} & 0.74 & 1.22 & $7.27 * *$ & $11.77^{* *}$ & $5.47 * *$ & 0.44 & 1.51 & 0.36 & 1.41 \\
\hline & \multicolumn{2}{|l|}{$\mathrm{C} * \mathrm{~T}$} & 0.17 & 2.29 & $9.38^{* *}$ & $4.87 *$ & 2.91 & 0.81 & 1.69 & 1.38 & 0.63 \\
\hline
\end{tabular}


Table 4. Effects of transplanting conditions and water management on proline content in leaves of fragrant rice $(\mu \mathrm{g} / \mathrm{g})$

\begin{tabular}{|c|c|c|c|c|c|c|}
\hline Season & Cultivar & Treatment & Tillering stage & Booting stage & Heading stage & $\begin{array}{c}\text { Heading after } \\
15 \mathrm{~d}\end{array}$ \\
\hline \multirow{8}{*}{ Early } & \multirow{4}{*}{ Meixiangzhan 2} & DR & $38.11 \pm 1.29 \mathrm{a}$ & $41.81 \pm 0.84 a$ & $31.77 \pm 1.91 \mathrm{~b}$ & $20.49 \pm 2.49 a$ \\
\hline & & DC & $40.33 \pm 1.86 \mathrm{a}$ & $52.43 \pm 3.72 \mathrm{a}$ & $28.71 \pm 1.14 b$ & $12.76 \pm 2.62 b c$ \\
\hline & & WR & $29.79 \pm 1.63 b$ & $37.52 \pm 7.22 b$ & $24.65 \pm 1.26 \mathrm{c}$ & $17.21 \pm 1.62 \mathrm{ab}$ \\
\hline & & WC & $30.79 \pm 1.22 b$ & $34.07 \pm 7.55 b$ & $40.35 \pm 1.41 \mathrm{a}$ & $11.83 \pm 0.84 c$ \\
\hline & \multirow{4}{*}{ Xiangyaxiangzhan } & DR & $30.68 \pm 2.88 \mathrm{a}$ & $35.39 \pm 3.54 a$ & $26.27 \pm 1.38 \mathrm{a}$ & $9.51 \pm 1.06 \mathrm{~b}$ \\
\hline & & DC & $32.11 \pm 4.66 \mathrm{a}$ & $38.41 \pm 4.18 \mathrm{a}$ & $26.31 \pm 1.72 \mathrm{a}$ & $5.29 \pm 0.51 \mathrm{c}$ \\
\hline & & WR & $17.13 \pm 5.21 \mathrm{c}$ & $19.12 \pm 7.48 b$ & $21.73 \pm 2.07 \mathrm{ab}$ & $14.71 \pm 1.64 \mathrm{a}$ \\
\hline & & WC & $21.22 \pm 6.33 \mathrm{~b}$ & $24.01 \pm 0.81 \mathrm{~b}$ & $19.43 \pm 3.37 \mathrm{~b}$ & $3.01 \pm 1.11 \mathrm{c}$ \\
\hline \multirow{8}{*}{ Late } & \multirow{4}{*}{ Meixiangzhan 2} & DR & $50.73 \pm 4.66 \mathrm{a}$ & $58.51 \pm 3.66 a$ & $36.92 \pm 0.61 \mathrm{a}$ & $79.13 \pm 2.68 \mathrm{a}$ \\
\hline & & DC & $50.66 \pm 2.54 \mathrm{a}$ & $52.51 \pm 1.33 a$ & $31.69 \pm 1.33 b$ & $51.36 \pm 3.49 b$ \\
\hline & & WR & $42.49 \pm 1.33 c$ & $34.98 \pm 1.92 \mathrm{c}$ & $26.13 \pm 2.57 \mathrm{c}$ & $42.52 \pm 1.43 c$ \\
\hline & & WC & $44.51 \pm 3.05 b$ & $46.62 \pm 4.39 b$ & $25.06 \pm 4.13 c$ & $31.73 \pm 0.82 \mathrm{~d}$ \\
\hline & \multirow{4}{*}{ Xiangyaxiangzhan } & $\overline{\mathrm{DR}}$ & $53.54 \pm 0.91 \mathrm{a}$ & $38.84 \pm 2.33 a$ & $20.48 \pm 0.85 a$ & $33.48 \pm 3.45 a$ \\
\hline & & DC & $40.97 \pm 4.48 b$ & $22.67 \pm 2.23 b$ & $14.35 \pm 2.07 \mathrm{~b}$ & $20.98 \pm 4.15 b c$ \\
\hline & & WR & $30.44 \pm 1.02 \mathrm{c}$ & $22.03 \pm 0.77 b$ & $15.48 \pm 2.41 b$ & $26.93 \pm 4.54 \mathrm{ab}$ \\
\hline & & WC & $36.09 \pm 4.31 b$ & $18.52 \pm 3.13 b$ & $12.76 \pm 0.39 b$ & $20.43 \pm 0.74 c$ \\
\hline
\end{tabular}

As shown in Table 5, for Meixiangzhan 2, the trend of proline content in stem was similar to that in leaves in the early season. There was no remarkable difference among the four stages. But tillering stage, the content of proline in DR and DC treatments were significantly higher than in WR and WC treatments. For Xiangyaxiangzhan, the content of proline in DR and DC treatments were significantly higher than in WR and WC treatments among the four stages in the early season and the trend in the late season was similar.

Table 5. Effects of transplanting conditions and water management on proline content in the stem of fragrant rice $(\mu \mathrm{g} / \mathrm{g})$

\begin{tabular}{|c|c|c|c|c|c|c|}
\hline Season & Cultivar & Treatment & Tillering stage & Booting stage & Heading stage & $\begin{array}{c}\text { Heading after } \\
\text { 15d } \\
\end{array}$ \\
\hline \multirow{8}{*}{ Early } & \multirow{4}{*}{ Meixiangzhan 2} & $\overline{\mathrm{DR}}$ & $24.13 \pm 4.02 \mathrm{a}$ & $16.12 \pm 2.09 \mathrm{~b}$ & $9.45 \pm 0.42 \mathrm{a}$ & $11.26 \pm 0.99 \mathrm{ab}$ \\
\hline & & DC & $15.99 \pm 1.14 \mathrm{~b}$ & $21.36 \pm 0.66 a$ & $8.45 \pm 0.89 b c$ & $14.17 \pm 3.70 \mathrm{a}$ \\
\hline & & WR & $12.42 \pm 1.37 \mathrm{c}$ & $9.98 \pm 0.71 \mathrm{c}$ & $9.71 \pm 1.22 \mathrm{~b}$ & $9.48 \pm 1.02 b c$ \\
\hline & & WC & $12.65 \pm 1.17 \mathrm{c}$ & $13.12 \pm 1.75 b c$ & $4.47 \pm 0.40 \mathrm{c}$ & $5.24 \pm 0.70 \mathrm{c}$ \\
\hline & \multirow{4}{*}{ Xiangyaxiangzhan } & DR & $15.09 \pm 1.21 \mathrm{a}$ & $11.83 \pm 1.25 \mathrm{a}$ & $3.68 \pm 0.75 a$ & $5.59 \pm 3.04 a$ \\
\hline & & DC & $16.28 \pm 3.77 \mathrm{a}$ & $11.25 \pm 1.02 \mathrm{a}$ & $2.98 \pm 1.09 \mathrm{a}$ & $4.03 \pm 1.77 \mathrm{ab}$ \\
\hline & & WR & $7.66 \pm 1.66 \mathrm{~b}$ & $6.71 \pm 1.97 \mathrm{~b}$ & $3.34 \pm 1.14 \mathrm{a}$ & $0.98 \pm 0.34 c$ \\
\hline & & WC & $11.82 \pm 2.3 \mathrm{ab}$ & $3.44 \pm 0.61 \mathrm{c}$ & $3.31 \pm 0.36 \mathrm{a}$ & $2.53 \pm 0.14 \mathrm{~b}$ \\
\hline \multirow{8}{*}{ Late } & \multirow{4}{*}{ Meixiangzhan 2} & DR & $38.53 \pm 4.44 \mathrm{a}$ & $23.38 \pm 1.46 b$ & $13.62 \pm 1.05 \mathrm{a}$ & $19.86 \pm 3.82 b$ \\
\hline & & DC & $33.23 \pm 3.57 \mathrm{a}$ & $24.71 \pm 3.6 \mathrm{ab}$ & $14.58 \pm 2.16 \mathrm{a}$ & $26.91 \pm 2.20 \mathrm{a}$ \\
\hline & & WR & $23.31 \pm 1.63 b$ & $23.62 \pm 4.15 b$ & $16.30 \pm 1.20 \mathrm{a}$ & $13.40 \pm 2.77 \mathrm{c}$ \\
\hline & & WC & $27.59 \pm 2.98 \mathrm{~b}$ & $29.94 \pm 2.67 \mathrm{a}$ & $16.82 \pm 3.45 \mathrm{a}$ & $24.31 \pm 2.49 \mathrm{ab}$ \\
\hline & \multirow{4}{*}{ Xiangyaxiangzhan } & DR & $25.49 \pm 3.91 \mathrm{a}$ & $18.91 \pm 3.11 \mathrm{a}$ & $11.14 \pm 2.42 \mathrm{a}$ & $15.84 \pm 1.44 \mathrm{a}$ \\
\hline & & DC & $19.39 \pm 2.37 \mathrm{~b}$ & $6.57 \pm 2.66 \mathrm{bc}$ & $7.08 \pm 3.27 \mathrm{ab}$ & $10.36 \pm 1.32 b$ \\
\hline & & WR & $14.83 \pm 2.11 \mathrm{c}$ & $7.18 \pm 1.33 b$ & $5.52 \pm 1.27 \mathrm{~b}$ & $5.02 \pm 1.79 c$ \\
\hline & & WC & $18.55 \pm 2.05 \mathrm{~b}$ & $3.05 \pm 0.56 \mathrm{c}$ & $4.11 \pm 0.85 b$ & $3.30 \pm 2.38 c$ \\
\hline
\end{tabular}




\section{Ornithine aminotransferase activities}

As shown in Table 6, for Meixiangzhan 2, the highest Ornithine aminotransferase (OAT) activity was recorded in DC treatment from heading stage to 15 days after heading (except WC treatment at heading stage) in the early season. OAT activity in the leaves of this cultivar under WR treatment was the highest at four stages (except heading stage) in the late season. For Xiangyaxiangzhan, compared with $\mathrm{C}$ treatments, $\mathrm{R}$ treatments enhanced OAT activity in leaves from tillering stage to booting stage.

Table 6. Effects of transplanting conditions and water management on OAT activity in the leaves of fragrant rice $(U \cdot g-1 \cdot \min -1 \cdot F W)$

\begin{tabular}{|c|c|c|c|c|c|c|}
\hline Season & Cultivar & Treatments & Tillering stage & Booting stage & Heading stage & Heading after $15 d$ \\
\hline \multirow{8}{*}{ Early } & \multirow{4}{*}{ Meixiangzhan 2} & DR & $115.77 \pm 3.66 \mathrm{a}$ & $114.58 \pm 4.75 \mathrm{a}$ & $71.72 \pm 1.43 b$ & $81.23 \pm 8.83 \mathrm{ab}$ \\
\hline & & DC & $113.87 \pm 3.44 \mathrm{a}$ & $110.96 \pm 1.12 \mathrm{a}$ & $76.05 \pm 1.63 \mathrm{a}$ & $89.07 \pm 7.66 a$ \\
\hline & & WR & $111.29 \pm 1.04 \mathrm{a}$ & $114.87 \pm 3.77 \mathrm{a}$ & $71.78 \pm 1.15 b$ & $74.70 \pm 1.76 b$ \\
\hline & & WC & $110.69 \pm 4.64 \mathrm{a}$ & $110.63 \pm 1.53 \mathrm{a}$ & $74.88 \pm 0.87 \mathrm{a}$ & $73.41 \pm 1.40 \mathrm{~b}$ \\
\hline & \multirow{4}{*}{ Xiangyaxiangzhan } & DR & $113.31 \pm 4.12 \mathrm{a}$ & $110.72 \pm 2.04 \mathrm{a}$ & $73.16 \pm 0.69 b$ & $75.82 \pm 2.83 a$ \\
\hline & & DC & $112.23 \pm 3.13 \mathrm{ab}$ & $107.47 \pm 7.87 \mathrm{~b}$ & $74.66 \pm 0.97 b$ & $73.52 \pm 2.64 \mathrm{a}$ \\
\hline & & WR & $111.59 \pm 2.00 \mathrm{ab}$ & $112.08 \pm 1.73 \mathrm{a}$ & $74.35 \pm 2.18 b$ & $74.26 \pm 2.64 \mathrm{a}$ \\
\hline & & WC & $105.34 \pm 3.82 \mathrm{~b}$ & $113.27 \pm 1.71 \mathrm{a}$ & $85.62 \pm 12.20 \mathrm{a}$ & $73.57 \pm 0.70 \mathrm{a}$ \\
\hline \multirow{8}{*}{ Late } & \multirow{4}{*}{ Meixiangzhan 2} & DR & $97.97 \pm 0.99 b$ & $94.39 \pm 1.30 \mathrm{a}$ & $87.90 \pm 1.70 \mathrm{a}$ & $82.99 \pm 1.39 a$ \\
\hline & & DC & $94.47 \pm 0.58 \mathrm{c}$ & $88.98 \pm 3.10 b$ & $86.25 \pm 0.98 \mathrm{a}$ & $82.56 \pm 1.60 \mathrm{a}$ \\
\hline & & WR & $101.20 \pm 1.28 \mathrm{a}$ & $94.47 \pm 2.91 \mathrm{a}$ & $85.64 \pm 2.35 \mathrm{a}$ & $83.33 \pm 2.85 a$ \\
\hline & & WC & $94.51 \pm 0.92 \mathrm{c}$ & $86.21 \pm 2.79 b$ & $84.25 \pm 4.73 a$ & $82.33 \pm 0.84 a$ \\
\hline & \multirow{4}{*}{ Xiangyaxiangzhan } & DR & $96.53 \pm 2.22 \mathrm{a}$ & $89.12 \pm 1.01 \mathrm{ab}$ & $82.72 \pm 2.12 \mathrm{a}$ & $81.90 \pm 2.21 \mathrm{ab}$ \\
\hline & & DC & $102.07 \pm 4.53 \mathrm{a}$ & $87.83 \pm 1.46 b$ & $83.65 \pm 4.94 a$ & $86.33 \pm 1.30 \mathrm{a}$ \\
\hline & & WR & $99.25 \pm 2.51 \mathrm{a}$ & $87.86 \pm 3.03 b$ & $85.35 \pm 1.82 \mathrm{a}$ & $84.02 \pm 2.6 \mathrm{ab}$ \\
\hline & & WC & $97.03 \pm 1.78 \mathrm{a}$ & $92.71 \pm 2.51 \mathrm{a}$ & $83.32 \pm 4.52 \mathrm{a}$ & $81.04 \pm 3.32 b$ \\
\hline
\end{tabular}

As shown in Table 7, for Meixiangzhan 2, the highest OAT activity in stems was recorded under the WC treatment at tillering stage and full heading stage in the early season, and the stems of OAT activity under DC treatment was the highest in the late season except for booting stage and 15 days after heading. For Xiangyaxiangzhan, the trend of OAT activity in the early season was the same as that of Meixiangzhan 2 , and the OAT activity was highest under WC treatment at 15 days after heading in the late season.

\section{Pyrroline-5-carboxylic acid content}

As shown in Table 8, for Meixiangzhan 2, the highest content of Pyrroline-5carboxylic acid (P5C) in leaves was recorded under the WR treatment at four stages of the early season (except heading after 15 days). Compared with WC and DC treatments, DR and WR treatments could significantly increase the content of P5C in leaves of this cultivar in the late season. For Xiangyaxiangzhan, in the early season, compared with WC and DC treatments, WR and DR treatments could increase P5C content at tillering stage and booting stage, and P5C content under WR treatment was significantly higher than that of other treatments (except WC treatment) at tillering stage in the late season. There was no significant difference among the treatments in the other three periods. 
Table 7. Effects of transplanting conditions and water management on OAT activity in the stem of fragrant rice $(U \cdot g-1 \cdot \mathrm{min}-1 \cdot F W)$

\begin{tabular}{|c|c|c|c|c|c|c|}
\hline Season & Cultivar & Treatment & Tillering stage & Booting stage & Heading stage & Heading after $15 d$ \\
\hline \multirow{8}{*}{ Early } & \multirow{4}{*}{ Meixiangzhan 2} & DR & $110.21 \pm 0.73 b$ & $113.11 \pm 3.75 \mathrm{a}$ & $72.69 \pm 2.95 a$ & $74.29 \pm 2.01 \mathrm{a}$ \\
\hline & & $\mathrm{DC}$ & $109.94 \pm 2.83 b$ & $114.05 \pm 3.36 \mathrm{a}$ & $71.63 \pm 1.54 \mathrm{ab}$ & $73.51 \pm 0.41 \mathrm{a}$ \\
\hline & & WR & $112.20 \pm 4.58 \mathrm{ab}$ & $108.65 \pm 0.76 b$ & $70.61 \pm 0.67 b$ & $72.66 \pm 1.03 \mathrm{a}$ \\
\hline & & WC & $115.59 \pm 0.71 \mathrm{a}$ & $112.80 \pm 1.25 \mathrm{a}$ & $74.38 \pm 1.41 \mathrm{a}$ & $73.13 \pm 2.70 \mathrm{a}$ \\
\hline & \multirow{4}{*}{ Xiangyaxiangzhan } & DR & $111.18 \pm 0.68 \mathrm{a}$ & $113.62 \pm 3.37 \mathrm{a}$ & $71.47 \pm 0.72 b$ & $73.53 \pm 2.06 b$ \\
\hline & & $\mathrm{DC}$ & $109.81 \pm 1.40 \mathrm{a}$ & $110.13 \pm 4.57 b$ & $71.27 \pm 2.81 b$ & $76.05 \pm 1.55 \mathrm{a}$ \\
\hline & & WR & $110.02 \pm 3.26 \mathrm{a}$ & $114.86 \pm 1.41 \mathrm{a}$ & $70.23 \pm 0.72 b$ & $73.51 \pm 2.53 b$ \\
\hline & & WC & $112.61 \pm 5.26 a$ & $113.46 \pm 4.20 \mathrm{a}$ & $74.03 \pm 1.69 a$ & $73.93 \pm 2.54 \mathrm{ab}$ \\
\hline \multirow{8}{*}{ Late } & \multirow{4}{*}{ Meixiangzhan 2} & DR & $80.74 \pm 3.11 b$ & $87.19 \pm 2.25 \mathrm{a}$ & $90.44 \pm 4.38 \mathrm{a}$ & $93.27 \pm 4.02 \mathrm{a}$ \\
\hline & & $\mathrm{DC}$ & $83.65 \pm 2.36 \mathrm{a}$ & $83.01 \pm 2.68 b$ & $91.07 \pm 4.02 \mathrm{a}$ & $88.59 \pm 0.81 b$ \\
\hline & & WR & $82.31 \pm 4.19 a$ & $84.96 \pm 3.00 \mathrm{~b}$ & $89.79 \pm 3.02 \mathrm{a}$ & $91.47 \pm 2.88 \mathrm{a}$ \\
\hline & & WC & $82.38 \pm 3.18 \mathrm{a}$ & $84.30 \pm 3.20 \mathrm{~b}$ & $88.63 \pm 3.82 \mathrm{a}$ & $92.87 \pm 4.17 \mathrm{a}$ \\
\hline & \multirow{4}{*}{ Xiangyaxiangzhan } & DR & $80.98 \pm 3.64 a$ & $80.53 \pm 3.43 b$ & $88.67 \pm 0.70 \mathrm{a}$ & $89.47 \pm 4.68 \mathrm{ab}$ \\
\hline & & $\mathrm{DC}$ & $79.52 \pm 4.62 \mathrm{a}$ & $85.17 \pm 3.35 \mathrm{a}$ & $87.47 \pm 2.26 \mathrm{a}$ & $88.26 \pm 3.66 \mathrm{ab}$ \\
\hline & & WR & $82.86 \pm 2.95 \mathrm{a}$ & $86.43 \pm 2.52 \mathrm{a}$ & $86.42 \pm 2.45 a$ & $87.77 \pm 4.90 b$ \\
\hline & & WC & $83.31 \pm 2.50 \mathrm{a}$ & $81.31 \pm 3.57 b$ & $88.30 \pm 1.36 \mathrm{a}$ & $93.16 \pm 1.84 a$ \\
\hline
\end{tabular}

Table 8. Effects of transplanting conditions and water management on the content of P5C in the leaves of fragrant rice $(\mathrm{u} \mathrm{mol} / \mathrm{g} \cdot \mathrm{FW})$

\begin{tabular}{|c|c|c|c|c|c|c|}
\hline Season & Cultivar & Treatment & Tillering stage & Booting stage & Heading stage & Heading after 15d \\
\hline \multirow{8}{*}{ Early } & \multirow{4}{*}{ Meixiangzhan 2} & DR & $1.48 \pm 0.02 b$ & $1.70 \pm 0.06 \mathrm{a}$ & $1.96 \pm 0.01 \mathrm{ab}$ & $2.09 \pm 0.10 \mathrm{ab}$ \\
\hline & & DC & $1.44 \pm 0.15 b$ & $1.55 \pm 0.08 \mathrm{~b}$ & $2.04 \pm 0.04 \mathrm{ab}$ & $2.23 \pm 0.11 \mathrm{a}$ \\
\hline & & WR & $1.52 \pm 0.02 \mathrm{a}$ & $1.71 \pm 0.10 \mathrm{a}$ & $2.07 \pm 0.08 \mathrm{a}$ & $2.20 \pm 0.05 a$ \\
\hline & & WC & $1.58 \pm 0.04 \mathrm{a}$ & $1.58 \pm 0.05 b$ & $1.95 \pm 0.01 b$ & $1.97 \pm 0.01 b$ \\
\hline & \multirow{4}{*}{ Xiangyaxiangzhan } & DR & $1.65 \pm 0.02 \mathrm{ab}$ & $1.56 \pm 0.02 \mathrm{a}$ & $1.68 \pm 0.02 b$ & $2.21 \pm 0.06 \mathrm{a}$ \\
\hline & & DC & $1.42 \pm 0.07 b$ & $1.42 \pm 0.04 \mathrm{~b}$ & $1.63 \pm 0.01 b$ & $2.12 \pm 0.09 \mathrm{a}$ \\
\hline & & WR & $1.72 \pm 0.05 \mathrm{a}$ & $1.54 \pm 0.02 \mathrm{a}$ & $1.69 \pm 0.01 b$ & $1.72 \pm 0.02 \mathrm{c}$ \\
\hline & & WC & $1.47 \pm 0.19 b$ & $1.48 \pm 0.03 b$ & $1.75 \pm 0.04 \mathrm{a}$ & $1.92 \pm 0.09 b$ \\
\hline \multirow{8}{*}{ Late } & \multirow{4}{*}{ Meixiangzhan 2} & $\mathrm{DR}$ & $1.80 \pm 0.02 \mathrm{a}$ & $2.24 \pm 0.04 \mathrm{a}$ & $2.12 \pm 0.06 b$ & $2.84 \pm 0.12 \mathrm{a}$ \\
\hline & & DC & $1.66 \pm 0.08 \mathrm{a}$ & $2.15 \pm 0.06 \mathrm{a}$ & $2.30 \pm 0.1 \mathrm{ab}$ & $2.86 \pm 0.17 \mathrm{a}$ \\
\hline & & WR & $1.72 \pm 0.04 \mathrm{a}$ & $2.23 \pm 0.14 \mathrm{a}$ & $2.38 \pm 0.13 \mathrm{a}$ & $2.57 \pm 0.17 b$ \\
\hline & & WC & $1.63 \pm 0.26 \mathrm{a}$ & $2.20 \pm 0.15 a$ & $2.15 \pm 0.11 b$ & $2.38 \pm 0.05 b$ \\
\hline & \multirow{4}{*}{ Xiangyaxiangzhan } & DR & $1.71 \pm 0.06 \mathrm{~b}$ & $2.07 \pm 0.06 \mathrm{a}$ & $2.08 \pm 0.08 \mathrm{a}$ & $2.68 \pm 0.23 a$ \\
\hline & & $\mathrm{DC}$ & $1.67 \pm 0.04 b$ & $1.99 \pm 0.06 \mathrm{a}$ & $2.11 \pm 0.05 \mathrm{a}$ & $2.43 \pm 0.37 \mathrm{a}$ \\
\hline & & WR & $1.84 \pm 0.06 b$ & $2.07 \pm 0.07 \mathrm{a}$ & $2.22 \pm 0.09 \mathrm{a}$ & $2.55 \pm 0.19 \mathrm{a}$ \\
\hline & & WC & $1.87 \pm 0.09 \mathrm{a}$ & $2.07 \pm 0.11 \mathrm{a}$ & $2.16 \pm 0.08 \mathrm{a}$ & $2.55 \pm 0.06 a$ \\
\hline
\end{tabular}

As shown in Table 9, for Meixiangzhan 2, the content of P5C in the stem of Meixiangzhan 2 was the highest under WC treatment at four stages in the early season, and the highest content of P5C in the stem of this cultivar was recorded in WR treatment at tillering stage and 15 days after heading in the late season. For Xiangyaxiangzhan, there is no significant difference among the four treatments in the early season, but the content of P5C in WC treatment is the highest at tillering stage and heading stage. 
Table 9. Effects of transplanting conditions and water management on the content of P5C in the stem of fragrant rice (umol/g.FW)

\begin{tabular}{c|c|c|c|c|c|c}
\hline Season & Cultivar & Treatment & Tillering stage & Booting stage & Heading stage & Heading after 15d \\
\hline \multirow{6}{*}{ Early } & DR & $1.01 \pm 0.09 \mathrm{a}$ & $1.15 \pm 0.01 \mathrm{~b}$ & $1.19 \pm 0.01 \mathrm{~b}$ & $1.21 \pm 0.02 \mathrm{~b}$ \\
& \multirow{4}{*}{ Meixiangzhan 2 } & DC & $1.01 \pm 0.02 \mathrm{a}$ & $1.14 \pm 0.01 \mathrm{bc}$ & $1.27 \pm 0.02 \mathrm{a}$ & $1.29 \pm 0.02 \mathrm{a}$ \\
& & WR & $1.03 \pm 0.03 \mathrm{a}$ & $1.12 \pm 0.01 \mathrm{c}$ & $1.21 \pm 0.02 \mathrm{~b}$ & $1.32 \pm 0.05 \mathrm{a}$ \\
& & WC & $1.08 \pm 0.05 \mathrm{a}$ & $1.18 \pm 0.01 \mathrm{a}$ & $1.32 \pm 0.03 \mathrm{a}$ & $1.28 \pm 0.02 \mathrm{a}$ \\
\cline { 2 - 7 } & \multirow{3}{*}{ Xiangyaxiangzhan } & DR & $1.01 \pm 0.02 \mathrm{a}$ & $1.13 \pm 0.02 \mathrm{a}$ & $1.26 \pm 0.09 \mathrm{a}$ & $1.20 \pm 0.03 \mathrm{a}$ \\
& & DC & $1.01 \pm 0.07 \mathrm{a}$ & $1.14 \pm 0.01 \mathrm{a}$ & $1.21 \pm 0.06 \mathrm{a}$ & $1.17 \pm 0.03 \mathrm{a}$ \\
& & WR & $0.98 \pm 0.01 \mathrm{a}$ & $1.17 \pm 0.01 \mathrm{a}$ & $1.18 \pm 0.03 \mathrm{~b}$ & $1.17 \pm 0.02 \mathrm{a}$ \\
& & WC & $1.08 \pm 0.03 \mathrm{a}$ & $1.14 \pm 0.04 \mathrm{a}$ & $1.20 \pm 0.03 \mathrm{a}$ & $1.15 \pm 0.01 \mathrm{a}$ \\
\hline & \multirow{3}{*}{ Late } & DR & $1.42 \pm 0.05 \mathrm{~b}$ & $1.19 \pm 0.03 \mathrm{a}$ & $1.44 \pm 0.05 \mathrm{a}$ & $1.46 \pm 0.06 \mathrm{~b}$ \\
& & DC & $1.18 \pm 0.03 \mathrm{c}$ & $1.26 \pm 0.06 \mathrm{a}$ & $1.36 \pm 0.08 \mathrm{a}$ & $1.58 \pm 0.04 \mathrm{a}$ \\
& & WR & $1.73 \pm 0.04 \mathrm{a}$ & $1.27 \pm 0.08 \mathrm{a}$ & $1.39 \pm 0.04 \mathrm{a}$ & $1.65 \pm 0.05 \mathrm{a}$ \\
& & WC & $1.39 \pm 0.05 \mathrm{~b}$ & $1.18 \pm 0.01 \mathrm{a}$ & $1.42 \pm 0.08 \mathrm{a}$ & $1.59 \pm 0.08 \mathrm{a}$ \\
\cline { 3 - 7 } & \multirow{3}{*}{ Xiangyaxiangzhan 2 } & DR & $1.39 \pm 0.19 \mathrm{~b}$ & $1.16 \pm 0.05 \mathrm{~b}$ & $1.27 \pm 0.02 \mathrm{~b}$ & $1.48 \pm 0.01 \mathrm{~b}$ \\
& & DC & $1.57 \pm 0.06 \mathrm{~b}$ & $1.24 \pm 0.02 \mathrm{a}$ & $1.30 \pm 0.01 \mathrm{~b}$ & $1.70 \pm 0.08 \mathrm{a}$ \\
& & WR & $1.41 \pm 0.06 \mathrm{~b}$ & $1.20 \pm 0.04 \mathrm{ab}$ & $1.23 \pm 0.01 \mathrm{c}$ & $1.56 \pm 0.10 \mathrm{~b}$ \\
& & WC & $1.89 \pm 0.14 \mathrm{a}$ & $1.22 \pm 0.04 \mathrm{ab}$ & $1.36 \pm 0.02 \mathrm{a}$ & $1.43 \pm 0.07 \mathrm{~b}$ \\
\hline
\end{tabular}

\section{Methylglyoxal content}

As shown in Table 10, for Meixiangzhan 2, the methylglyoxal content in the leaves of this cultivar under DC treatment was the highest at booting stage in the early season, and there was no significant difference in methylglyoxal content among the treatments except for DR treatment at full heading stage. From tillering stage to heading stage in the late season, WR treatment maximized content of methylglyoxal in leaves of this cultivar. For Xiangyaxiangzhan, in the tillering stage of the early season, the content of methylglyoxal was the highest recorded under the WR treatment.

Table 10. Effects of transplanting conditions and water management on methylglyoxal in the leaves of fragrant rice $(\mu \mathrm{mol} / \mathrm{g} \cdot \mathrm{FW})$

\begin{tabular}{|c|c|c|c|c|c|c|}
\hline Season & Cultivar & Treatment & Tillering stage & Booting stage & Heading stage & Heading after 15d \\
\hline \multirow{8}{*}{ Early } & \multirow{4}{*}{ Meixiangzhan 2} & DR & $21.24 \pm 0.37 \mathrm{c}$ & $21.15 \pm 0.73 b$ & $23.54 \pm 0.45 b$ & $24.17 \pm 1.54 \mathrm{a}$ \\
\hline & & $\mathrm{DC}$ & $25.09 \pm 0.43 a$ & $21.95 \pm 0.75 b$ & $28.12 \pm 1.74 \mathrm{a}$ & $22.25 \pm 0.89 a$ \\
\hline & & WR & $23.76 \pm 0.40 b$ & $20.60 \pm 0.34 b$ & $26.27 \pm 0.37 \mathrm{a}$ & $22.26 \pm 2.33 \mathrm{a}$ \\
\hline & & WC & $21.74 \pm 0.39 c$ & $24.53 \pm 0.72 \mathrm{a}$ & $26.13 \pm 0.71 \mathrm{a}$ & $21.43 \pm 0.27 \mathrm{a}$ \\
\hline & \multirow{4}{*}{ Xiangyaxiangzhan } & DR & $24.50 \pm 2.23 b$ & $22.42 \pm 0.20 \mathrm{a}$ & $22.08 \pm 0.93 \mathrm{a}$ & $21.53 \pm 1.04 \mathrm{a}$ \\
\hline & & DC & $27.64 \pm 2.21 \mathrm{ab}$ & $19.90 \pm 0.56 b$ & $22.05 \pm 1.51 \mathrm{a}$ & $22.21 \pm 1.10 \mathrm{a}$ \\
\hline & & WR & $30.76 \pm 1.42 \mathrm{a}$ & $21.44 \pm 1.05 \mathrm{a}$ & $22.10 \pm 1.09 \mathrm{a}$ & $17.64 \pm 1.17 b$ \\
\hline & & $\mathrm{WC}$ & $26.15 \pm 0.56 b$ & $21.66 \pm 0.56 \mathrm{a}$ & $22.79 \pm 1.75 \mathrm{a}$ & $20.13 \pm 0.76 \mathrm{a}$ \\
\hline \multirow{8}{*}{ Late } & \multirow{4}{*}{ Meixiangzhan 2} & $\overline{D R}$ & $23.39 \pm 0.44 b c$ & $21.66 \pm 0.60 \mathrm{~b}$ & $24.63 \pm 0.90 \mathrm{a}$ & $31.02 \pm 1.92 \mathrm{a}$ \\
\hline & & DC & $22.40 \pm 2.19 c$ & $19.92 \pm 1.30 \mathrm{~b}$ & $24.81 \pm 0.39 \mathrm{a}$ & $28.23 \pm 1.78 \mathrm{a}$ \\
\hline & & WR & $26.01 \pm 0.93 a$ & $23.85 \pm 1.40 \mathrm{a}$ & $26.01 \pm 1.02 \mathrm{a}$ & $28.24 \pm 0.17 \mathrm{a}$ \\
\hline & & $\mathrm{WC}$ & $25.59 \pm 1.02 \mathrm{ab}$ & $21.22 \pm 1.11 \mathrm{~b}$ & $22.26 \pm 1.22 b$ & $28.38 \pm 1.48 \mathrm{a}$ \\
\hline & \multirow{4}{*}{ Xiangyaxiangzhan } & DR & $24.98 \pm 0.37 \mathrm{ab}$ & $24.34 \pm 0.79 a$ & $25.94 \pm 0.74 b$ & $26.95 \pm 0.66 \mathrm{a}$ \\
\hline & & $\mathrm{DC}$ & $23.83 \pm 0.32 b$ & $21.22 \pm 1.40 \mathrm{~b}$ & $26.48 \pm 0.33 \mathrm{ab}$ & $26.87 \pm 1.87 \mathrm{a}$ \\
\hline & & WR & $25.55 \pm 0.77 \mathrm{a}$ & $21.62 \pm 1.29 b$ & $25.57 \pm 1.17 b$ & $27.65 \pm 2.08 \mathrm{a}$ \\
\hline & & WC & $25.83 \pm 1.13 \mathrm{a}$ & $24.45 \pm 2.98 \mathrm{a}$ & $27.43 \pm 0.55 \mathrm{a}$ & $26.93 \pm 0.64 a$ \\
\hline
\end{tabular}


As shown in Table 11, for Meixiangzhan 2, the highest content of methylglyoxal in the stem was recorded under the DR treatment at 15 days after heading in the early season. For Xiangyaxiangzhan, there were no remarkable trends in the early season. And in the late season, the content of methylglyoxal trend was: $\mathrm{DR}=\mathrm{WR}>\mathrm{WC}>\mathrm{DC}$.

Table 11. Effects of transplanting conditions and water management on methylglyoxal in the stem of fragrant rice $(\mathrm{mol} / \mathrm{g} \cdot \mathrm{FW})$

\begin{tabular}{|c|c|c|c|c|c|c|}
\hline Season & Cultivar & Treatment & Tillering stage & Booting stage & Heading stage & Heading after 15d \\
\hline \multirow{8}{*}{ Early } & \multirow{4}{*}{ Meixiangzhan 2} & DR & $14.40 \pm 0.61 \mathrm{a}$ & $14.46 \pm 0.24 \mathrm{a}$ & $12.64 \pm 0.52 \mathrm{ab}$ & $16.77 \pm 0.33 a$ \\
\hline & & DC & $14.37 \pm 0.34 \mathrm{a}$ & $14.96 \pm 0.50 \mathrm{a}$ & $12.13 \pm 0.23 b$ & $15.67 \pm 0.08 \mathrm{ab}$ \\
\hline & & WR & $14.45 \pm 0.34 \mathrm{a}$ & $14.74 \pm 0.69 \mathrm{a}$ & $11.93 \pm 0.01 b$ & $14.81 \pm 0.35 b$ \\
\hline & & WC & $14.60 \pm 0.50 \mathrm{a}$ & $15.23 \pm 0.28 \mathrm{a}$ & $13.08 \pm 0.51 \mathrm{a}$ & $14.73 \pm 1.20 \mathrm{~b}$ \\
\hline & \multirow{4}{*}{ Xiangyaxiangzhan } & DR & $13.14 \pm 0.52 \mathrm{a}$ & $12.44 \pm 0.04 \mathrm{a}$ & $11.81 \pm 0.28 \mathrm{ab}$ & $14.39 \pm 0.53 b$ \\
\hline & & DC & $13.38 \pm 0.88 \mathrm{a}$ & $13.23 \pm 1.27 \mathrm{a}$ & $12.28 \pm 0.55 \mathrm{a}$ & $15.74 \pm 0.10 \mathrm{a}$ \\
\hline & & WR & $13.11 \pm 0.24 \mathrm{a}$ & $13.94 \pm 0.60 \mathrm{a}$ & $11.75 \pm 0.49 \mathrm{ab}$ & $14.95 \pm 0.8 \mathrm{ab}$ \\
\hline & & WC & $13.06 \pm 0.64 \mathrm{a}$ & $13.73 \pm 1.29 \mathrm{a}$ & $10.33 \pm 1.49 b$ & $15.85 \pm 0.42 \mathrm{a}$ \\
\hline \multirow{8}{*}{ Late } & \multirow{4}{*}{ Meixiangzhan 2} & DR & $13.95 \pm 0.65 \mathrm{ab}$ & $11.29 \pm 1.16 \mathrm{~b}$ & $13.18 \pm 0.58 \mathrm{ab}$ & $14.63 \pm 0.18 \mathrm{a}$ \\
\hline & & DC & $13.17 \pm 0.60 \mathrm{~b}$ & $12.75 \pm 0.41 \mathrm{a}$ & $12.03 \pm 0.73 b$ & $15.27 \pm 0.33 \mathrm{a}$ \\
\hline & & WR & $13.51 \pm 0.33 \mathrm{ab}$ & $13.63 \pm 0.8 \mathrm{a}$ & $13.91 \pm 0.51 \mathrm{a}$ & $14.86 \pm 0.71 \mathrm{a}$ \\
\hline & & WC & $14.28 \pm 0.44 \mathrm{a}$ & $12.97 \pm 0.36 \mathrm{a}$ & $12.41 \pm 1.36 \mathrm{ab}$ & $15.31 \pm 0.88 \mathrm{a}$ \\
\hline & \multirow{4}{*}{ Xiangyaxiangzhan } & DR & $13.25 \pm 0.44 \mathrm{a}$ & $13.01 \pm 0.22 \mathrm{a}$ & $12.54 \pm 1.54 \mathrm{a}$ & $15.79 \pm 0.73 b$ \\
\hline & & DC & $12.66 \pm 0.54 \mathrm{ab}$ & $13.03 \pm 0.30 \mathrm{a}$ & $12.78 \pm 0.22 \mathrm{a}$ & $16.78 \pm 0.35 \mathrm{a}$ \\
\hline & & WR & $13.13 \pm 0.30 \mathrm{a}$ & $13.55 \pm 0.89 \mathrm{a}$ & $12.45 \pm 0.51 \mathrm{a}$ & $16.21 \pm 0.60 \mathrm{ab}$ \\
\hline & & WC & $11.95 \pm 0.13 b$ & $13.81 \pm 0.07 \mathrm{a}$ & $12.57 \pm 0.17 \mathrm{a}$ & $16.15 \pm 0.08 \mathrm{ab}$ \\
\hline
\end{tabular}

\section{2-acetyl-1-pyrroline content in grains}

As shown in Table 12, for Meixiangzhan 2, at the mature stage of the early season, the trend of 2-Acetyl-1-Pyrroline (2-AP) content was: $\mathrm{WR}=\mathrm{DR}>\mathrm{WC}>\mathrm{DC}$. But at the mature stage of late season the trend of 2-AP was: $\mathrm{WC}>\mathrm{WR}=\mathrm{DC}>\mathrm{DR}$. For Xiangyaxiangzhan, the trend of grain aroma content in mature stage was consistent with that of Meixiangzhan 2, and compared with $\mathrm{C}$ treatments, the average aroma content of $\mathrm{R}$ treatments was increased by $5.24 \%$ in the early season, and the highest aroma at mature stage was recorded under the WR treatment in the late season.

\section{Discussion}

Various yield components act comprehensively and coordinate with each other to form the yield of rice. Water management, as an important measure to increase yield, has been concerned by predecessors. With the decrease of irrigation water, the number of effective panicles of direct seeding rice increased at first and then decreased (Wu et al., 2019). Water-saving irrigation could increase the number of tillers in the middle and early stage, also it increased the number of effective tillers in the later stage as well as the panicle rate of tillers (Zhao et al., 2018). Light dry-wet alternative irrigation can effectively control ineffective tillers, and significantly increased panicle rate (Zhao et al., 2015). The results showed that the fertile panicle number of the two fragrant rice had no significant difference between WR and WC treatment, but were higher than DR and DC treatments which prove that water-saving irrigation maybe not only saving the resource but also can enhance or keep the fertile panicle number. Under the condition of 
alternating dry and wet irrigation, it was helpful to the construction of high yield population, the coordination of nitrogen accumulation and distribution of rice plant (Peng, 2014). The results showed that the highest grain count per panicle, seed setting rate and 1000-grain weight of two fragrant rice cultivars were recorded in WR treatment (except WC treatment) which means that WR treatment was more beneficial to the export and accumulation of assimilates during rice growth, and made the yield composition more reasonable. On the other hand, compared to conventional irrigation treatment in WC treatment no extra water was applied in the rice production, thus WR treatment is beneficial to water conservation. We observed that there was no significant difference between WR and WC treatment of average yield in both seasons. Thus, WR treatment could be a water-saving cultivation.

Table 12. Effects of transplanting conditions and water management on the aroma (2-AP) content of fragrant rice seeds

\begin{tabular}{|c|c|c|c|c|}
\hline Season & Cultivar & Treatment & HA 15d & Maturity stage \\
\hline \multirow{8}{*}{ Early } & \multirow{4}{*}{ Meixiangzhan 2} & DR & $258.44 \pm 5.77 \mathrm{bc}$ & $252.02 \pm 5.4 \mathrm{a}$ \\
\hline & & DC & $251.39 \pm 8.02 \mathrm{c}$ & $169.60 \pm 5.00 \mathrm{c}$ \\
\hline & & WR & $298.40 \pm 5.79 \mathrm{a}$ & $252.10 \pm 8.88 \mathrm{a}$ \\
\hline & & WC & $280.62 \pm 8.24 \mathrm{ab}$ & $230.56 \pm 5.85 b$ \\
\hline & \multirow{4}{*}{ Xiangyaxiangzhan } & $\mathrm{DR}$ & $152.77 \pm 4.19 \mathrm{~d}$ & $307.08 \pm 16.72 \mathrm{a}$ \\
\hline & & DC & $175.20 \pm 15.13 c$ & $245.19 \pm 4.49 b$ \\
\hline & & WR & $246.21 \pm 7.50 \mathrm{~b}$ & $301.06 \pm 15.65 \mathrm{a}$ \\
\hline & & WC & $276.11 \pm 3.65 \mathrm{a}$ & $286.36 \pm 12.95 \mathrm{ab}$ \\
\hline \multirow{8}{*}{ Late } & \multirow{4}{*}{ Meixiangzhan 2} & DR & $264.83 \pm 11.09 \mathrm{~b}$ & $140.65 \pm 6.85 c$ \\
\hline & & DC & $141.07 \pm 8.46 c$ & $174.52 \pm 10.54 b$ \\
\hline & & WR & $300.29 \pm 14.30 \mathrm{ab}$ & $181.79 \pm 9.61 b$ \\
\hline & & WC & $349.02 \pm 25.49 \mathrm{a}$ & $235.11 \pm 11.17 \mathrm{a}$ \\
\hline & \multirow{4}{*}{ Xiangyaxiangzhan } & DR & $252.17 \pm 4.39 c$ & $135.59 \pm 5.43 c$ \\
\hline & & DC & $254.88 \pm 5.13 \mathrm{c}$ & $232.88 \pm 14.71 b$ \\
\hline & & WR & $313.17 \pm 10.93 b$ & $265.59 \pm 8.48 \mathrm{a}$ \\
\hline & & WC & $363.78 \pm 22.33 \mathrm{a}$ & $232.03 \pm 3.27 b$ \\
\hline
\end{tabular}

Water stress at different stages had significant effects on the quality of rice, such as chalky grain rate, protein content and fat content. Water stress treatment from flowering to 15 days after anthesis significantly reduced the head rice rate of rice, but had relatively little effect on other quality factors (Zheng et al., 2017). The results showed that there was no significant difference in brown rice rate and milled rice rate between the two fragrant rice cultivars under different treatments, while the highest head rice rate was recorded in $\mathrm{R}$ treatments which proved that the processing quality of rice could be improved under this treatment. The milled rice rate and head rice rate were maximized in WR treatment, also minimized in chalkiness and chalky grain rate in all except WC treatments in the early season of Xiangyaxiangzhan, and the four treatments had little effect on the length-width ratio of the two fragrant rice cultivars.

As the aroma characteristic substance, 2-acetyl-1-pyrroline (2-AP) of fragrant rice can form pyrroline-5-carboxylic acid by proline as nitrogen source and catalyzed by proline oxidase. Ornithine aminotransferase can also catalyze ornithine to form 
pyrroline 5-carboxylic acid, decarboxylate to form 1-pyrroline, and finally combine with acetyl CoA. Under the action of pyrroline acetyltransferase to form the aroma of 2acetyl-1-pyrroline (Yoshihashi et al., 2002). This study showed that compared with other treatments, WR treatment increased P5C content in both early and late seasons, and the content of 2-AP in grains of two fragrant rice cultivars treated with WR was enhanced, indicating that WR treatment had the effect of increasing aroma.

\section{Conclusions}

The average yield of WR treatment was similar to WC treatment, since the varieties and the treatments did not influence the yield significantly. Furthermore, WR treatment had the highest milled rice rate and head rice rate and the lowest chalkiness and chalky grain rate all except WC treatments in the early season. WR treatment increased P5C content of two fragrant rice cultivars in both early and late seasons, and the content of 2AP in grains of two fragrant rice cultivars under WR treatment was enhanced. Hence, WR treatment had the effect of increasing aroma. In conclusion, WR treatment could be a water-saving cultivation, and the optimum treatment for increasing yield and improving quality, also it increases the aroma of fragrant rice grains. Although, further research is required at molecular and physiological level.

Acknowledgements. This study was supported by the Natural Science Foundation of China (31971843). The authors declare no conflicts of interests.

\section{REFERENCES}

[1] Abid, M., Khan, I., Mahmood, F., Ashraf, U., Imran, M., Anjum, S. A. (2015): Response of hybrid rice to various transplanting dates and nitrogen application rates. - Philippine Agricultural Scientist 98: 98-104.

[2] Bao, G., Ashraf, U., Wang, C., He, L., Wei, X., Zheng, A., Mo, Z., Tang, X. (2018): Molecular basis for increased 2-acetyl-1-pyrroline contents under alternate wetting and drying (AWD) conditions in fragrant rice. - Plant Physiology and Biochemistry 133: 149157.

[3] Deng, Q. Q., Ashraf, U., Cheng, S. R., Sabir, S. R., Mo, Z. W., Pan, S. G., Tian, H., Duan, M. Y., Tang, X. R. (2018): Mild drought in interaction with additional nitrogen dose at grain filling stage modulates 2acetyl-1-pyrroline biosynthesis and grain yield in fragrant rice. - Applied Ecology and Environmental Research 16: 7741-7758.

[4] Du, P., Luo, H., He, J., Mao, T., Du, B., Hu, L. (2019): Different tillage induces regulation in 2-acetyl-1-pyrroline biosynthesis in direct-seeded fragrant rice. - BMC Plant Biology 19.

[5] Graham Acquaah, S., Siebenmorgen, T. J., Reba, M. L., Massey, J. H., Mauromoustakos, A., Adviento Borbe, A., January, R., Burgos, R., Baltz Gray, J. (2019): Impact of alternative irrigation practices on rice quality. - Cereal Chemistry 96: 815-823.

[6] Hasanuzzaman, M., Alam, M. M., Nahar, K., Mohsin, S. M., Bhuyan, M. H. M. B., Parvin, K., Hawrylak-Nowak, B., Fujita, M. (2019): Silicon-induced antioxidant defense and methylglyoxal detoxification works coordinately in alleviating nickel toxicity in Oryza sativa L. - Ecotoxicology 28: 261-276.

[7] Malek, M. A., Latif, M. A. (2013): Biochemical, genetic and molecular advances of fragrance characteristics in rice. - Critical Reviews in Plant Sciences 32: 445-457. 
[8] Huang, Z. L., Tang, X. R., Wang, Y. L., Chen, M. J., Zhao, Z. K., Duan, M. Y., Pan, S. G. (2012): Effects of fragrant cultivation on aroma and yield of scented rice and its related physiological mechanism. - Chinese Agricultural Science 45: 1054-1065

[9] Kong, L., Luo, H., Mo, Z., Pan, S., Liu, Z., Zhang, Q., Bai, S., Tang, X. (2020): Grain yield, quality and 2-Acetyl-1-Pyrroline of fragrant rice in response to Different planting seasons in south china. - Phyton 89: 705-714.

[10] Luo, H., Du, B., He, L., He, J., Hu, L., Pan, S., Tang, X. (2019): Exogenous application of zinc (Zn) at the heading stage regulates 2-acetyl-1-pyrroline (2-AP) biosynthesis in different fragrant rice genotypes. - Scientific Reports 9: 19513.

[11] Marchesi, C., Chauhan, B. S. (2019): The efficacy of chemical options to control Echinochloa crus-galli in dry-seeded rice under alternative irrigation management and field layout. - Crop Protection 118: 72-78.

[12] Peng, Y. (2014): Effects of seedling age, water management and slow/controlled release fertilizer on nitrogen use characteristics and yield formation of rice. - Master Thesis, Sichuan Agricultural University, Ya'an.

[13] Tao, H. B., Lin, S. (2006): Comparison of hole weighing method with copy weighing method and length-width correction method for determining leaf area of rice. - Plant Physiology Newsletter 2006: 496-498.

[14] Tian, H., Pan, S. G., Mo, Z. W., Duan, M. Y., Tang, X. R. (2018): Effects of different water and grain fertilizer treatments on aroma, quality and yield of scented rice. - Journal of Irrigation and Drainage 37: 36-41.

[15] Wang, P., Tang, X. R., Tian, H., Pan, S. G., Duan, M. Y., Nie, J., Luo, Y. M., Xiao, L. Z. (2013): Effects of water irrigation treatments at booting stage on aroma and physiological characteristics of scented rice. - Guangdong Agricultural Sciences 40: 1-3.

[16] Wu, Y. X., Liu, F. Y., Sun, Y. J., Guo, C. C., Jerry Young, Yan, T. R., Sun, Z. B., Ding, F., Ma, L. (2019): Effects of interaction of water and nitrogen on yield and quality of direct seeding rice. - Journal of Sichuan Agricultural University 37: 604-610, 622.

[17] Huong, N. T. T., Inatomi, H. (2002): Precursors of 2-acetyl-1-pyrroline, a potent flavor compound of an aromatic rice variety. - Journal of Agricultural and Food Chemistry 50: 2001-2004.

[18] Zhao, L. M., Li, M., Zheng, D. F., Gu, C. M., Na, Y. G., Xie, B. S. (2015): Effects of irrigation method and planting density on rice yield and photosynthetic matter production characteristics in cold region. - Journal of Agricultural Engineering 2015: 159-169.

[19] Zhao, H. J., Wang, Q., Sun, Y., Zeng, X. N., Zhang, X. M., Wang, P., Wang, M. L., Feng, Y. J. (2018): Effects of straw returning irrigation on rice yield and water use efficiency. Journal of Nuclear Agriculture 32: 959-969.

[20] Zheng, C. J., Li, S. (2017): Effects of water stress on rice growth and rice quality during anthesis. - China Rice 23: 43-45. 


\section{APPENDIX}

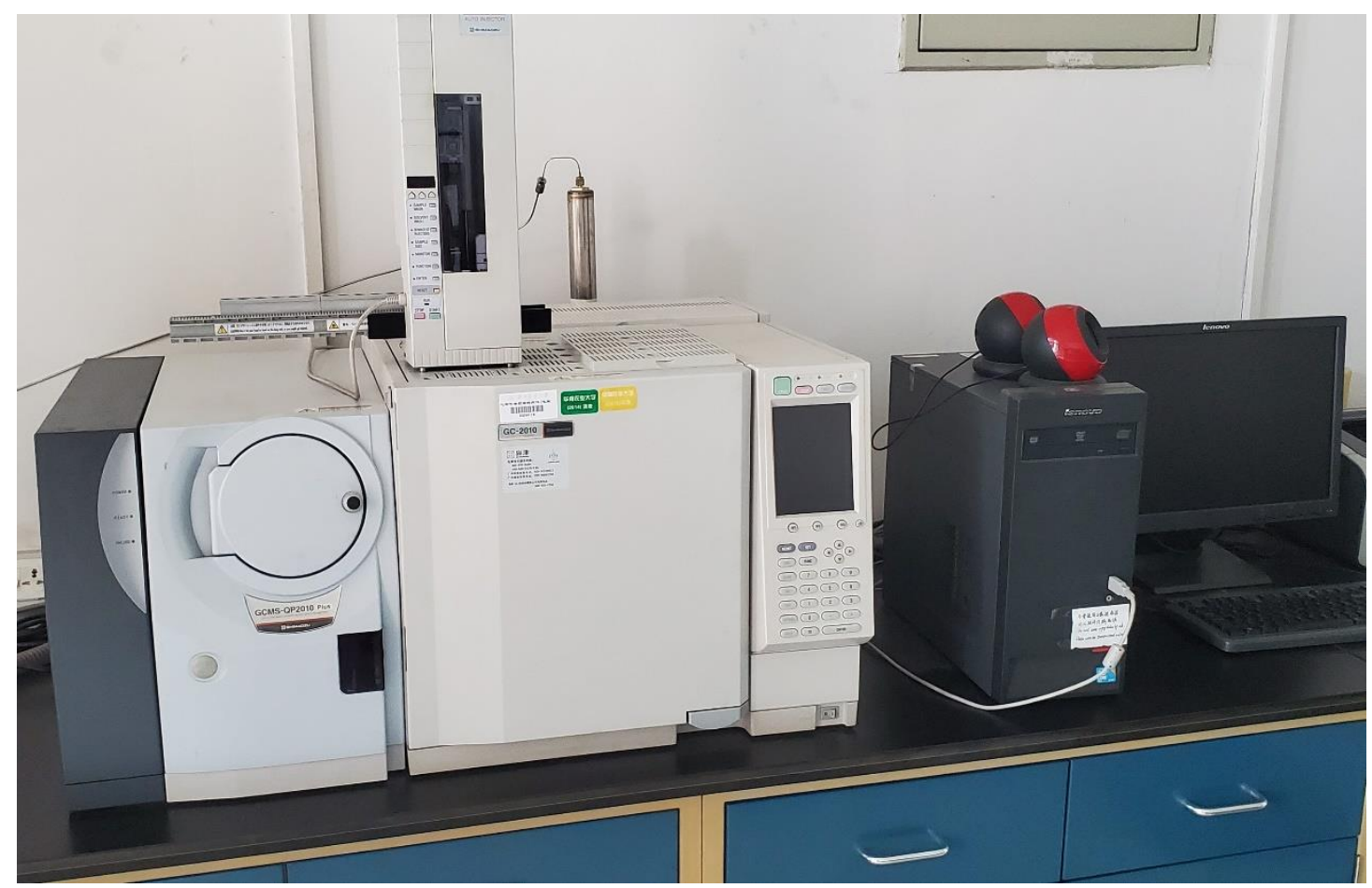

Instruments used to measure the content of aroma in fragrant rice 